\title{
Understanding the distribution, degradation and fate of organophosphate esters in an advanced municipal sewage treatment plant based on mass flow and mass balance analysis
}

\author{
Kang Liang a , Jingfu Liu ${ }^{\text {a,b,* }}$ \\ a State Key Laboratory of Environmental Chemistry and Ecotoxicology, Research Center for Eco-Environmental Science, Chinese Academy of Sciences, Beijing 100085, China \\ b Institute of Environment and Health, Jianghan University, Wuhan, Hubei Province 430056, China
}

\section{H I G H L I G H T S}

- Mass flow and mass balance are used to study the behaviors and fate of OPEs in STP

- Activated sludge system plays an effective role in removing OPEs from raw sewage

- The behavior and fate of individual OPE showed a polarity-specific feature.

- Sorption of OPEs to suspended solids was resulted from hydrophobic interactions

- OPE degradation in activated sludge system is more relevant to molecular structure

\section{G R A P H I C A L A B S T R A C T}

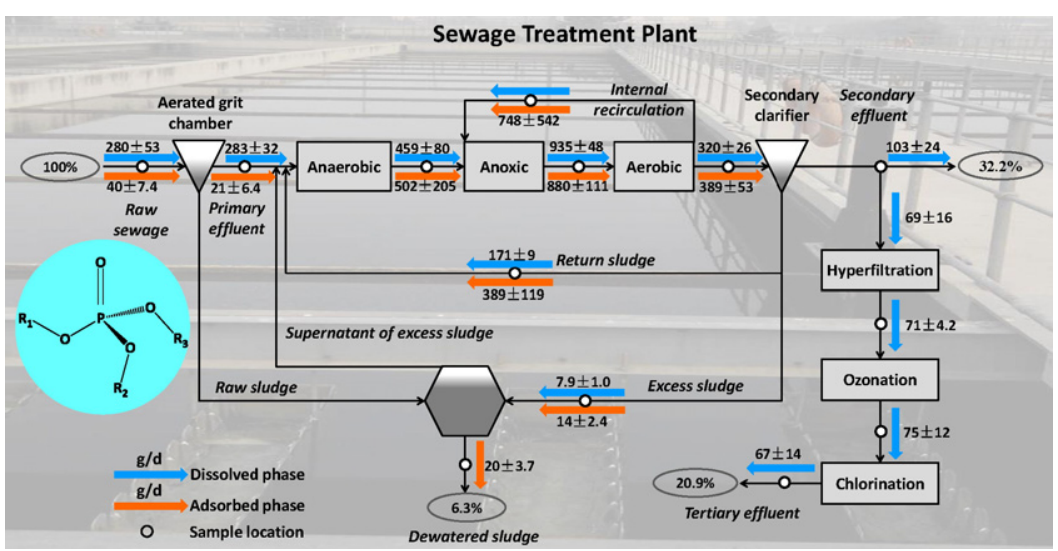

\section{A R T I C L E I N F O}

\section{Article history:}

Received 25 September 2015

Received in revised form 21 November 2015

Accepted 23 November 2015

Available online 3 December 2015

Editor: Adrian Covaci

\section{Keywords:}

Organophosphorus flame retardants

Wastewater

Mass balance analysis

Behavior

Fate

\footnotetext{
* Corresponding author.

E-mail address: jfliu@rcees.ac.cn (J. Liu).
}

\section{A B S T R A C T}

Although organophosphate esters (OPEs) in the ambient environment are from sewage treatment plants due to the discharge of effluent and application of sludge, the distribution, degradation and fate of OPEs in advanced municipal sewage treatment plants remain unclear. This work focused on the use of mass flow and mass balance analysis to understand the behaviors and fate of 14 OPEs in an advanced municipal sewage treatment plant. OPEs were detected in all sewage water and sludge samples with total OPEs ( $\mathrm{OPPEs}$ ) concentrations of $1399 \pm 263 \mathrm{ng} / \mathrm{L}$ in raw sewage aqueous phase, $833 \pm 175 \mathrm{ng} / \mathrm{L}$ in tertiary effluent aqueous phase, and $315 \pm 89 \mathrm{ng} / \mathrm{g}$ dry weight in dewatered sludge. The dissolved concentrations of $\Sigma$ OPEs significantly decreased during biological treatment, whereas negligible decrease was observed in mechanical and physical-chemical treatments. For individual OPE, the chlorinated tris(2-chloroethyl) phosphate (TCEP) and tris(2-chloroisopropyl) phosphate (TCPP) did not decrease but increased during both biological treatment and physical-chemical treatment. Mass flow analysis indicated the total removal efficiency of $\Sigma$ OPEs in aqueous phase was $40.5 \%$, and the polarity-specific removal efficiencies for individual OPE were positively related to their solid-water partition coefficients $\left(K_{\mathrm{d}}\right)$. Furthermore, mass balance results showed that $53.1 \%$ and $6.3 \%$ of the initial OPE mass flow were eventually transferred to the effluents and dewatered sludge, respectively, while the remaining $39.9 \%$ and $0.7 \%$ were lost due to biodegradation and physical-chemical treatment, respectively. It was indicated that the 
activated sludge treatment system with anaerobic/anoxic/aerobic bioreactors was a major factor in the removal of OPEs from the raw sewage, while transfer to dewatered sludge governed by hydrophobic interactions was limited during the sewage treatment. Meanwhile, the degradation difference of OPEs in activated sludge treatment was more related with their molecular structure over their hydrophobicity.

(c) 2015 Elsevier B.V. All rights reserved.

\section{Introduction}

Organophosphate esters (OPEs), one of the most commonly used organophosphorus flame retardants (OPFR) (van der Veen and de Boer, 2012), have been applied as flame retardant and plasticizer in plastics, electronic equipment, furniture, textiles, construction and transportation all over the world (Marklund et al., 2003; Reemtsma et al., 2008; Wang et al., 2010). Owing to the ban of some polybrominated diphenyl esters (Leung et al., 2007), the global consumption of alternative OPFR is under a rapid increase, up to 292,000 metric tons in 2011 from 198,000 metric tons in 2007 (Retardants-Online).

Along with their widespread production and application, OPEs can be slowly released into the environment by volatilization, leaching and abrasion (Sundkvist et al., 2010). As a result, they are now widely present in various environmental matrices worldwide, including water (Wang et al., 2011; Rodil et al., 2005; Marklund et al., 2005), soil (Fries and Mihajlovic, 2011; Mihajlovic et al., 2011), air (Moller et al., 2011; Moller et al., 2012), sediment (Cristale and Lacorte, 2013) and biota (Kim et al., 2011). Moreover, OPEs have already been detected in human milk (Sundkvist et al., 2010) and their metabolites were also found in human urine (van den Eede et al., 2013; Reemtsma et al., 2011; Schindler et al., 2009). However, due to the main toxic effects including eye and skin irritation, carcinogenicity, dermatitis, and neurotoxicity (Moller et al., 2012; van den Eede et al., 2012), OPEs were reported to show potential risk to human health. Given that, the frequent detection in environment and underlying threat for human health of OPEs call for insight studies on their transfer, transformation and fate in the environment.

At the same time, sewage water and sludge are the important media for OPEs to enter various environmental matrices. OPEs can directly get into the aquatic environment along with the sewage effluent (Fries and Puttmann, 2003), and enter the soil when the dewatered sludge are used as fertilizer in agriculture (Bester, 2005), resulting in water and soil contamination gradually. Therefore, the elimination of OPEs in sewage treatment plants (STPs) plays an important role in their pollution control.

Current studies mainly focused on the detection of OPEs in sewage water (Rodil et al., 2005; Garcia-Lopez et al., 2008; Martinez-Carballo et al., 2007; Ellis et al., 2007; Rodriguez et al., 2006; Quintana and Reemtsma, 2006) and sludge (Bester, 2005; Chen and Bester, 2009; Zeng et al., 2014), as well as the elimination of OPEs from sewage water (Marklund et al., 2005; Meyer and Bester, 2004). Research conducted at STPs in Germany (Meyer and Bester, 2004) and Sweden (Marklund et al., 2005) showed great removal difference for various OPEs (Table 1) in aqueous phase during sewage treatment: chlorinated alkyl OPEs showed no obvious elimination, while alkyl OPEs (TMP, TnBP, TPhP and TBEP in Table 1) showed an elimination in the range of $20-100 \%$. However, the elimination of individual OPE is dependent on the properties of specific OPE species and STPs. While the mean elimination of TBEP in Sweden STPs (23\%) was much lower than that in German STPs ( 90\%), the mean elimination of TnBP in Sweden STPs ( 85\%) was higher than that in German STPs ( 60\%). Furthermore, it should be highlighted that the concentration of chlorinated OPEs (TCEP and TCPP) in sewage effluent water was higher than that in raw sewage water in both studies. Accordingly, understanding the elimination and behavior of individual OPE during the sewage treatment is of great importance for reducing their underlying environmental and ecological risk.

Few studies focusing on the elimination of OPEs during sewage treatment have been reported. Meyer and Bester examined the effect of filter tank and the combined effect of aeration tank and sedimentation tank in sewage treatment (Meyer and Bester, 2004). Marklund et al. studied the fate of OPEs during sewage treatment based on an estimated mass balance calculation (Marklund et al., 2005). For an advanced sewage treatment plant with typical sewage treatment procedures including primary treatment (screen, grit chamber and primary sedimentation), secondary treatment (activated sludge or biofilm

Table 1

Total mass flow loss percentage of OPEs (sum of dissolved and adsorbed) at different treatment units based on the mass flow in the inflow and outflow of individual treatment unit.

\begin{tabular}{|c|c|c|c|c|c|c|c|c|c|c|}
\hline \multirow[t]{2}{*}{ OPES } & \multirow[t]{2}{*}{ Abbreviation } & \multicolumn{5}{|c|}{ Traditional treatment (\%) } & \multicolumn{4}{|c|}{ Advanced treatment (\%) } \\
\hline & & Anaerobic $^{a}$ & Anoxic & Oxic. & Sec. Sdm. ${ }^{\text {b }}$ & $\sum \mathrm{TT}^{\mathrm{c}}$ & Ultrafiltration & Ozonation & Chlorination & $\sum A T^{d}$ \\
\hline Trimethyl phosphate & TMP & -11 & -8.2 & 13 & 12 & 23 & 1.3 & 27 & -23 & 11 \\
\hline Triethyl phosphate & TEP & 2.5 & 5.1 & 0.2 & 6.8 & 22 & 14 & -8.5 & 3.7 & 11 \\
\hline Tris(2-chloroethyl) phosphate & TCEP & 14 & 16 & -16 & -41 & -19 & -6.6 & -5.6 & 2.8 & -9.4 \\
\hline Tri-n-propyl phosphate & TPrP & - & - & - & - & & - & - & - & - \\
\hline Tris(2-chloroisopropyl) phosphate & ТСРP & 19 & 35 & -64 & -5.7 & -39 & 7.4 & -12 & -1.1 & -4.6 \\
\hline Tris(2-chloro-1-(chloromethyl)ethyl) phosphate & TDCP & -30 & 17 & -14 & 21 & 39 & 12 & -5.7 & -21 & -12 \\
\hline Triphenyl phosphate & TiBP & 31 & 2.5 & -12 & -23 & -7.4 & 30 & -37 & 52 & 54 \\
\hline Triisobutyl phosphate & $\mathrm{TPhP}$ & 12 & 12 & -7.9 & -16 & 29 & 44 & -56 & 79 & 82 \\
\hline Tri-n-butyl phosphate & TnBP & -23 & -10 & 16 & 28 & 71 & -67 & -30 & 52 & -3.3 \\
\hline Cresyl diphenyl phosphate & CDPP & - & - & - & - & & - & - & - & - \\
\hline Tri(2-butoxyethyl) phosphate & TBEP & -57 & 26 & 27 & 1.5 & 90 & -112 & 18 & 48 & 9.5 \\
\hline Tri-3-cresyl phosphate & $\mathrm{TCrP}$ & -8.3 & 27 & -33 & 3.9 & 61 & 50 & 69 & 80 & 97 \\
\hline 2-Ethylhexyl diphenyl phosphate & EHDPP & -24 & 18 & -0.5 & -24 & 64 & 100 & - & - & -3.7 \\
\hline Tri(2-ethylhexyl) phosphate & TEHP & -39 & 5.4 & 15 & -23 & -3.1 & - & - & - & - \\
\hline Total OPEs & $\sum$ OPES & -11 & 21 & -13 & -6.0 & 40 & -2.2 & -5.8 & 11 & 3.4 \\
\hline
\end{tabular}

- = data not available

a Mass flow loss percentage of anaerobic unit was calculated ignoring the mass flow in the supernatant of excess sludge which was negligible comparing to the mass flow of primary effluent and return sludge, considering the supernatant of excess sludge was not accessible for sampling.

b Secondary sedimentation.

c Total mass flow loss percentage of OPEs in traditional treatment

d Total mass flow loss percentage of OPEs in advanced treatment. 
process), and tertiary treatment (ultrafiltration, ozonation, chlorination and ultraviolet disinfection), it is still unknown which process plays a key role in the elimination of OPEs during sewage treatment.

In this study, we try to gain insights into the behavior and fate of OPEs in the typical municipal STP. We analyzed the OPE concentration in sewage water and sludge samples, collected along the whole sewage treatment process in an advanced municipal STP with anaerobic/anoxic/aerobic $(\mathrm{A} / \mathrm{A} / \mathrm{O})$ bioreactors as the core treatment units. More importantly, mass flow and mass balance analysis were performed to understand the transport and the potential removal mechanisms of these compounds inside the treatment units.

\section{Materials and methods}

\subsection{Flow scheme of sewage treatment plant}

The STP investigated in this work serves approximately 814,000 people in Beijing, China, with a total sewage treatment capability of $400,000 \mathrm{~m}^{3} /$ day, which is mainly from domestic sources. The flow scheme of the STP is shown in Fig. 1. The raw sewage first passes through a screen and an aerated grit chamber successively. The primary sludge was pumped into the dehydrating house, while the primary effluent was directed to the activated sludge system, which is comprised of the anaerobic/anoxic/aerobic $(\mathrm{A} / \mathrm{A} / \mathrm{O})$ bioreactors. After a secondary clarification step, $60 \%$ of the secondary effluent is directly discharged to a receiving river, while the other $40 \%$ of the secondary effluent was then pumped to an advanced tertiary treatment system including ultrafiltration, ozonation, and chlorination processes. After the advanced treatment, the tertiary effluent is used as irrigation water and landscape water. The hydraulic retention times in anaerobic tank, anoxic tank, aerobic tank and secondary sedimentation tank were $1.5,3,10.8$, and $8 \mathrm{~h}$, respectively, and the total solid retention time was about 20-25 days. After settling in the secondary clarifier, most of the activated sludge was returned to the anaerobic bioreactor, and the rest as excess sludge was conveyed to be dehydrated. After that, the dewatered sludge was carried away for final disposal.

\subsection{Sample collection and pretreatment}

To investigate the behavior and fate of OPEs in this STP, sampling was carried out along sewage treatment process under normal dry weather conditions on March 24, 25, 26, 2014, respectively. The sampling locations in the STP are also shown in Fig. 1. All samples were taken at the outlet of every treatment step including aerated grit chamber, anaerobic tank, anoxic tank, aerobic tank, secondary sedimentation tank, ultrafiltration tank, ozonation tank and chlorination tank. Meanwhile, internal recirculation sludge from aerobic tank to anoxic tank, returned sludge from secondary sedimentation tank to anaerobic tank, excess sludge and dewatered sludge were also sampled, but raw sludge from aerated grit chamber and supernatant of excess sludge were not collected as they were inaccessible for sampling. All the samples were consisted of aqueous phase (water) and solid phase (suspended solid particles), except dewatered sludge, ultrafiltration effluent, ozonation effluent and the tertiary effluent. Single $24 \mathrm{~h}$ composite water samples of raw sewage and primary, secondary and tertiary effluents were collected by using automatic samplers at a sampling interval of $2 \mathrm{~h}$. Other solid-liquid and dewatered sludge samples were obtained as time-proportional $24 \mathrm{~h}$ composite samples by combining four grab samples collected four times a day with sampling interval of $6 \mathrm{~h}$. During the sampling period, the sewage influent to the STP kept around $200,000 \mathrm{~m}^{3} /$ day. The water flow at each treatment unit and several regularly measured parameters (e.g., pH, DOC and TOC) are shown in Supporting information (Table S1).

All samples, except for dewatered sludge, ultrafiltration effluent, ozonation effluent and the tertiary effluent, were immediately separated to the aqueous phase (water) and solid phase (suspended solids) by centrifugation at $10,397 \mathrm{~g}$ for $7 \mathrm{~min}$ (3-18K centrifuge, Sigma, Germany) after sampling. Before analysis, aqueous samples were filtered through a $1.2 \mathrm{~mm}$ glass microfiber membrane GF/C (Whatman, Maidstone, UK) and stored at $4{ }^{\circ} \mathrm{C}$; while suspended solids were frozen within hours and further freeze-dried, then ground before sifting through a sieve with 100 meshes, and finally stored at $-20{ }^{\circ} \mathrm{C}$ in refrigerator. The OPEs in aqueous samples were analyzed according to the method reported in our previous paper (Liang et al., 2014), while those in suspended solids were determined by a method developed in this work. Briefly, for aqueous phase, the analytes were extracted from $100 \mathrm{~mL}$ filtered aqueous samples onto HLB cartridges ( $6 \mathrm{~mL}, 200 \mathrm{mg}$, Waters, Milford, USA), and eluted by $6 \mathrm{~mL}$ acetonitrile, then the extracts were concentrated to near dryness and redissolved in $1 \mathrm{~mL}$ of acetonitrile/water $(40: 60, v / v)$ before determination. For solid phase, the analytes were extracted from $0.5 \mathrm{~g}$ freeze-dried suspended solids onto Florisil cartridges ( $3 \mathrm{~mL}, 500 \mathrm{mg}$, Supelco, Bellefonte, USA), and eluted by $8 \mathrm{~mL}$ dichloromethane, then the extracts were concentrated to dryness and redissolved in $1 \mathrm{~mL}$ of acetonitrile/water (40:60, v/v) before

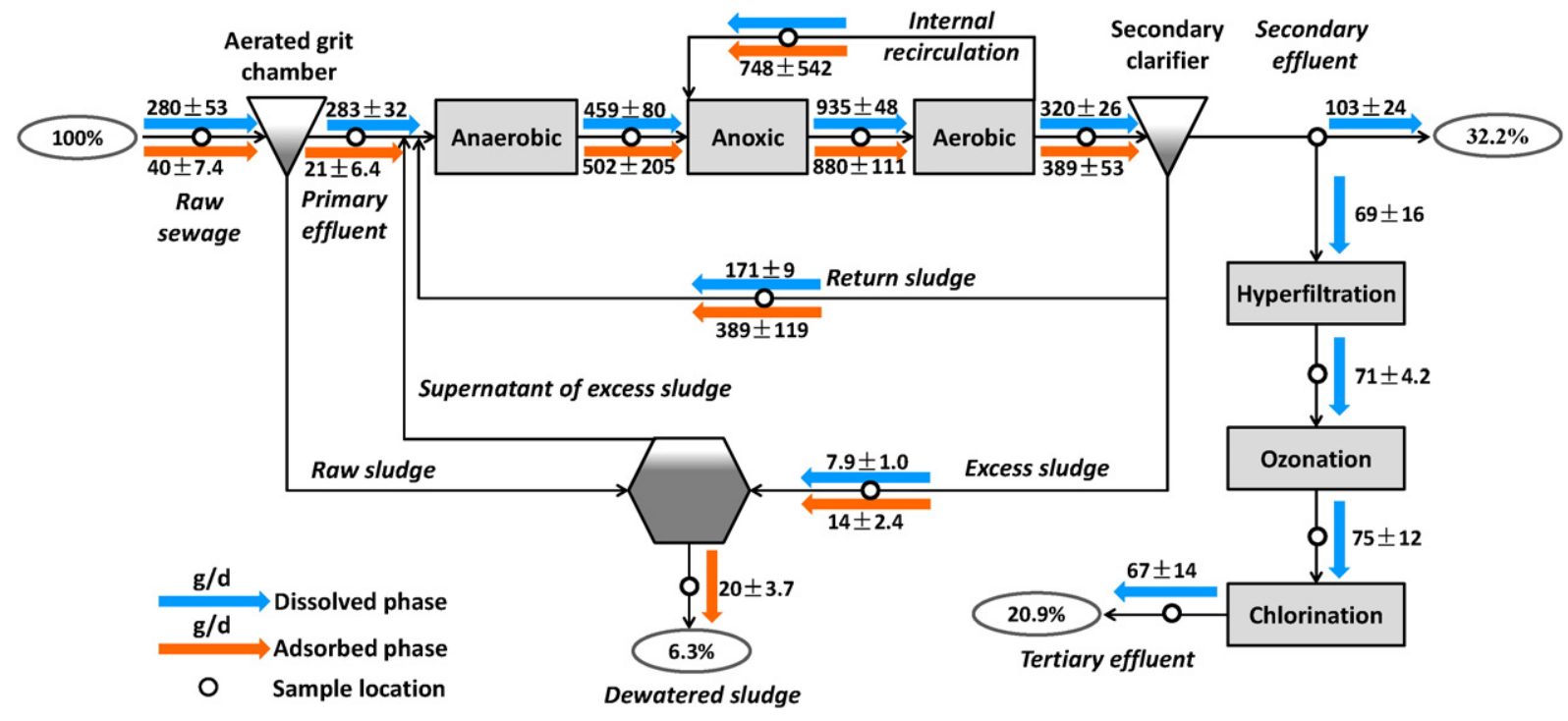

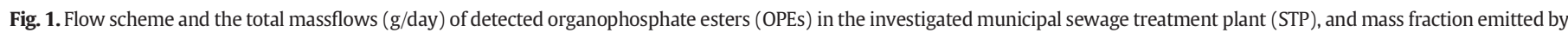
the plant in effluents and dewatered sludge relative to the total initial mass flow (100\%) of raw sewage. 
determination. Details about sample extraction and cleanup procedures of OPEs are given in Supporting information.

\subsection{Chemical analysis}

Using a UPLC apparatus (Acquity Ultra Performance LC, Waters, Milford, MA), 14 targeted OPEs were separated using a Waters Acquity UPLC BEH C18 column $(100 \times 2.1 \mathrm{~mm}, 1.7 \mu \mathrm{m}$ particle size $)$. The mobile phase was a mixture of $0.1 \%(\mathrm{v} / \mathrm{v})$ formic acid in ultrapure water $(\mathrm{A})$ and acetonitrile (B) with a flow rate of $0.2 \mathrm{~mL} / \mathrm{min}$ under gradient conditions: 0 min, 40\% B; 0.5 min, 40\% B; 3 min, 50\% B; 4.5 min, 55\% B; $8.5 \mathrm{~min}, 70 \% \mathrm{~B} ; 9 \mathrm{~min}, 100 \% \mathrm{~B} ; 13.8 \mathrm{~min}, 100 \% \mathrm{~B} ; 13.9 \mathrm{~min}, 40 \% \mathrm{~B}$; and $15.5 \mathrm{~min}, 40 \% \mathrm{~B}$. The injection volume was $10 \mu \mathrm{L}$ and the column was maintained at $40{ }^{\circ} \mathrm{C}$. Mass spectrometry determination was performed using a TSQ Quantum Access triple quadruple mass spectrometer (Thermo Scientific, Waltham, MA) equipped with an electrospray ionization (ESI) source. The ESI was operated in the positive ion mode, and best conditions were set as follows: spray voltage of $4000 \mathrm{~V}$, and capillary temperature of $300{ }^{\circ} \mathrm{C}$. Nitrogen was used as the sheath gas (35 units) and aux gas (15 units). Argon was used as the collision gas at a pressure of $0.2 \mathrm{~Pa}$. Detailed information on instrumental analysis can be found in our previous work (Liang et al., 2014).

\subsection{Quantification and quality control}

Method accuracy was evaluated by the recoveries of target analytes in aqueous samples and suspended solids, and the method precision was assessed by relative standard deviation (RSD). To correct the losses of analytes during extraction or sample preparation, and compensate for variations in instrument response from injection to injection, deuterated standards TMP-d9, TEP-d15, TPrP-d21 and TPhP-d15 were used as surrogate standards for TMP, TEP, TPrP and TPhP, respectively, while TnBP-d27 was for the other 10 OPEs. Spiked recoveries were determined by spiking at least three times the original concentration of standard OPEs into aqueous samples and solid samples. Quality control samples, consisting of spiked raw sewage aqueous sample, tertiary effluent water, and suspended solids collected from excess sludge, produced recoveries of $52.0-119 \%, 43.6-114 \%$ and $33.0-114 \%$, respectively. The limits of method detection (mLODs) for the target OPEs, defined as the minimum concentrations of compounds that could be measured (Table S2), ranged from 0.57 to $6.6 \mathrm{ng} / \mathrm{g}$ in the suspended solid samples, and from 0.30 to $5.7 \mathrm{ng} / \mathrm{L}$ in aqueous samples. Details of method performance are present in Supporting information (Table S2). For each set of samples at least one procedure blank and one independent check standard were run in sequence to check the background contamination and system performance.

\subsection{Mass balance calculation}

Mass balance was calculated by multiplying concentration of OPEs by average daily flow rates as shown in Eq. (1):

$W=C_{\text {dissolved }} \times Q+C_{\text {adsorbed }} \times Q \times C_{\text {TSS }}$

where $W(\mathrm{~g} / \mathrm{d})$ is the total mass flow of OPEs dissolved in aqueous phase and adsorbed to solid phase; $C_{\text {dissolved }}$ and $C_{\text {adsorbed }}$ represent the dissolved $(\mathrm{ng} / \mathrm{L})$ and adsorbed $(\mu \mathrm{g} / \mathrm{g})$ concentrations, respectively; $Q$ is water flow ( $\mathrm{m}^{3} /$ day); and $C_{\mathrm{TSS}}$ represents the content of total suspended solids (mg/L).

The mass flow loss of OPEs in individual treatment unit and mass flow loss percentage (removal efficiency) was calculated using Eq. (2) and Eq. (3), respectively:

$W_{\text {loss }}=W_{\text {inflow }}-W_{\text {outflow }}$

$\left(W_{\text {inflow }}-W_{\text {outflow }}\right) / W_{\text {inflow }} \times 100 \%$ where $W_{\text {inflow }}$ and $W_{\text {outflow }}$ represent the mass flow of OPEs in the inflow and outflow of individual treatment unit, respectively.

In order to assess the contribution of sorption and degradation of OPEs in the STP, the mass flow loss of OPEs owing to sum of all transfor-

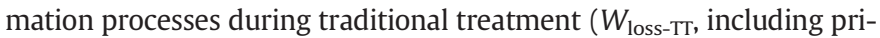
mary and secondary treatment) and advanced treatment process ( $W_{\text {loss-AT, }}$ tertiary treatment) was calculated using Eq. (4) and Eq. (5), respectively:

$W_{\text {loss-TT }}=W_{\text {raw }}-W_{\text {sec.eff. }}-W_{\text {sludge }}$

$W_{\text {loss-AT }}=0.4 \times W_{\text {sec.eff. }}-W_{\text {ter.eff. }}$

where $W_{\text {raw }}, W_{\text {sec. eff. and }} W_{\text {ter. eff. }}$ represent the mass flow of OPEs in raw sewage, secondary effluent and tertiary effluent (g/day), respectively; $W_{\text {sludge }}$ is the mass output of OPEs in dehydrated sludge (g/day).

\section{Results and discussion}

\subsection{Concentrations of OPEs in sewage water and sludge along treatment} process

OPEs were detected in either aqueous phase (dissolved, $\mathrm{ng} / \mathrm{L}$ ) or solid phase (adsorbed, ng/g d.w., d.w. refers to dry weight) of all samples collected through the STP. All the 14 target OPEs, except for TPrP, CDPP and TEHP, were detected in aqueous phase samples from the STP (Table S3). In raw sewage aqueous phase, TBEP (600 $\pm 173 \mathrm{ng} / \mathrm{L})$, TCPP $(225 \pm 23 \mathrm{ng} / \mathrm{L})$ and TCEP $(179 \pm 27 \mathrm{ng} / \mathrm{L})$ were the dominant OPEs, accounting for $42.9 \pm 12.4 \%, 16.1 \pm 1.7 \%, 12.8 \pm 1.9 \%$ of total concentrations, respectively (Fig. 2). Relatively low concentrations were detected in raw sewage aqueous phase for TEP (114 $\pm 54 \mathrm{ng} / \mathrm{L})$, TMP $(99 \pm 20 \mathrm{ng} / \mathrm{L}), \operatorname{TnBP}(74 \pm 12 \mathrm{ng} / \mathrm{L}), \mathrm{TiBP}(45 \pm 18 \mathrm{ng} / \mathrm{L}), \mathrm{TDCP}$ $(23 \pm 2 \mathrm{ng} / \mathrm{L}), \mathrm{TPhP}(21 \pm 5 \mathrm{ng} / \mathrm{L}), \mathrm{TCrP}(9 \pm 5 \mathrm{ng} / \mathrm{L})$ and EHDPP $(14 \pm 1 \mathrm{ng} / \mathrm{L})$. Of all the target compounds in this study, the predominance of TBEP, TCPP and TCEP were also observed in STP wastewater in most developed countries including Spain (Rodriguez et al., 2006; Quintana and Reemtsma, 2006; Garcia-Lopez et al., 2010), Germany (Rodil et al., 2005; Meyer and Bester, 2004), Austria (MartinezCarballo et al., 2007) and Sweden (Marklund et al., 2005) (Table 2). In the present study, raw sewage aqueous phase concentrations of TBEP, TCPP and TCEP were lower than those in surface water in Beijing: 10740 ng/L TBEP, 145-1359 ng/L TCPP, and 88.4-2918 ng/L TCEP (Gao et al., 2015), which may result from the separation of rain and sewage considering that rainwash can directly bring a plenty of OPEs from soil, dust and airborne particles into the river. Meanwhile, the raw sewage aqueous phase concentrations of these three OPEs were lower than those in most developed countries (Table 2), e.g. 680-3100 ng/L TBEP and 290-540 ng/L TCPP in Spain (Garcia-Lopez et al., 2010); 5200$35,000 \mathrm{ng} / \mathrm{L}$ TBEP, $1100-18,000 \mathrm{ng} / \mathrm{L}$ TCPP and 90-1000 ng/L TCEP in Sweden (Marklund et al., 2005); and 3700-8000 ng/L TBEP, 650$5800 \mathrm{ng} / \mathrm{L}$ TCPP and 180-640 ng/L TCEP in Germany (Meyer and Bester, 2004). Such a profile of OPEs in this STP is in accordance with the low consumption of these compounds in China compared with other countries. It was documented that the consumption of OPFRs in China was only $7 \%$ that of global consumption in 2008 , while the percentages of Europe and the United States were almost 40\% and 35\%, respectively (Posner, 2011). The total removal efficiencies of individual OPE in sewage water ranged from $-50.6 \%$ (TCPP) to $95.2 \%$ (TCrP) (Table 2). In tertiary effluent water, TCPP and TCEP, which showed no significant removal during the secondary treatment and tertiary treatment, were the dominant OPEs.

In the suspended solids and dewatered sludge samples, of all the 14 OPEs only TMP, TEP, TPrP and CDPP were not detected, i.e., the concentration of TEP was lower than that of procedural blank, while the concentrations of TMP, TPrP and CDPP were below the lower detection limit of method. The suspended particles in raw sewage showed the 


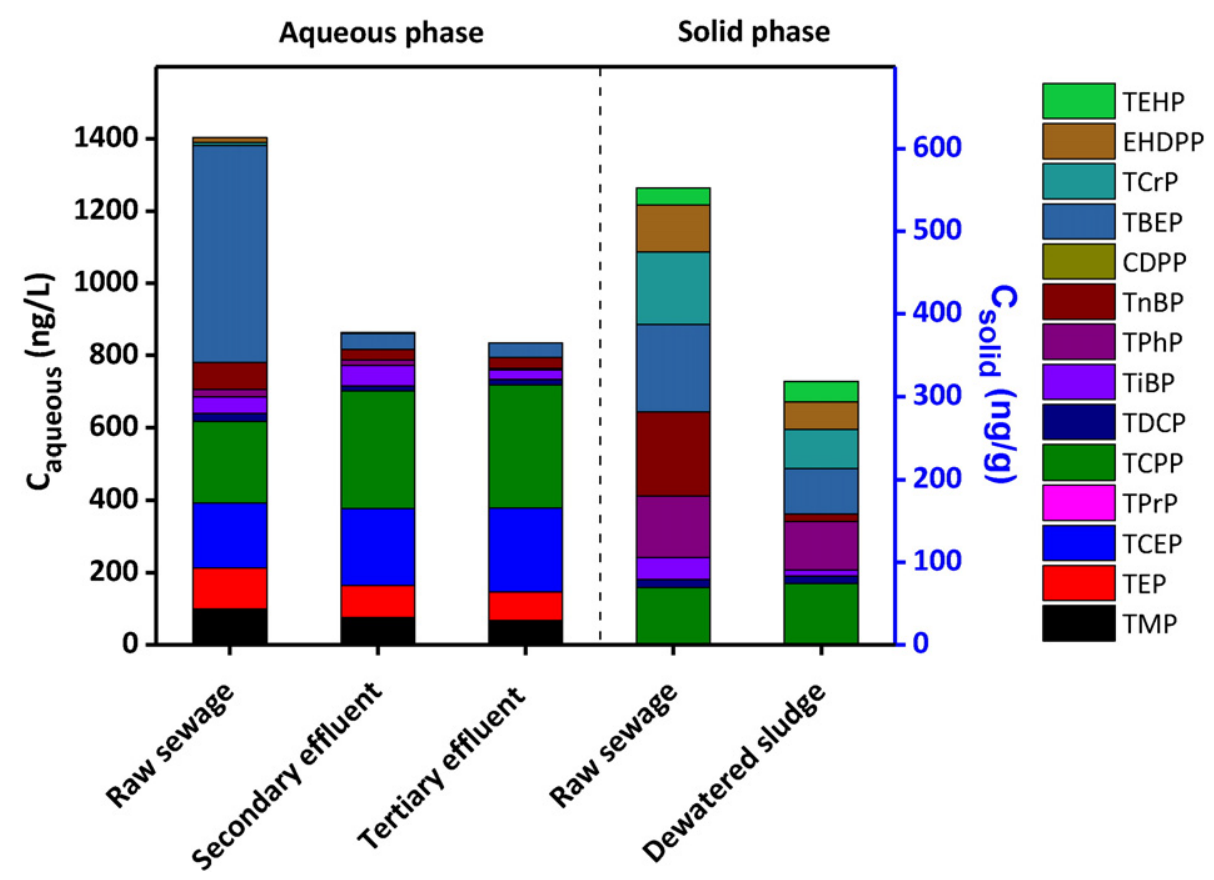

Fig. 2. Concentrations of OPEs in aqueous phase of raw sewage water, secondary effluent and tertiary effluent, and that in solid phase of raw sewage sludge and dewatered sludge.

highest levels of OPEs, with total adsorbed concentration up to $553 \pm$ $80 \mathrm{ng} / \mathrm{g}$, which was almost 2-fold higher than those of activated sludge in bioreactors. The adsorbed concentrations of OPEs in activated sludge along anaerobic/anoxic/aerobic (A/A/O) treatment units showed a slight decrease trend, which was from $337 \pm 147$ to $257 \pm 33 \mathrm{ng} / \mathrm{g}$ d.w. Both the return sludge and excess sludge showed a comparable concentration to that of activated sludge in aerobic tank. The measured adsorbed concentrations of OPEs in various types of sludge were similar, mainly because a large amount of activated sludge was constantly cycled as internal recirculation and return sludge in the STP. The concentration of OPEs in dewatered sludge was $315 \pm 89 \mathrm{ng} / \mathrm{g}$ d.w., which was equivalent to or slightly higher than those in activated sludge. The adsorbed concentrations of $\sum$ OPEs (214-553 ng/g d.w.) in the present study were higher than those of domestic wastewater in the Pearl River Delta, China (101-290 ng/g d.w.) (Zeng et al., 2014), but were lower than those of developed countries (620-6900 ng/g d.w. in Sweden, and 1000-20,000 ng/g d.w. in Germany) (Marklund et al., 2005; Bester, 2005; Chen and Bester, 2009).

\subsection{Mass flow analysis of OPEs during wastewater treatment}

To better understand the behavior like partitioning, mass flow and transfer of OPEs in STP, the calculated mass flows of $\sum$ OPEs in aqueous and solid phases through the whole sewage treatment process were presented in Fig. 1. Based on Fig. 1 and the mass flows of individual OPE (Table S4 and Table S5), we further analyzed mass flow loss percentages in the four basic treatment units and three advanced treatment units to assess their contributions and potential mechanism in removal of OPEs in the STP (Table 1).

In raw sewage, the mass flow of $\sum$ OPEs combined aqueous and solid phase was about $320 \mathrm{~g} /$ day in this STP, i.e. 280 and $40 \mathrm{~g} /$ day in the dissolved and adsorbed phase, respectively. The mass losses rate of OPEs across the aerated grit chamber was not available because of the absence of sample in raw sludge. After the aerated grit chamber, $304 \mathrm{~g} /$ day $\sum$ OPEs remaining in primary effluent entered secondary activated sludge system. The mass flow of aqueous phase in primary effluent and raw sewage were very similar, while the mass flow of suspended solids in primary effluent was $47.0 \%$ less than that in raw sewage (Table S6). This might be caused by the transfer of OPEs to raw sludge along with the suspended solids from raw sewage, in view of a $23.6 \%$ mass reduction (Table S6). As a result, the total mass flow of hydrophobic OPEs, mainly distributed in sludge, were largely decreased after primary treatment, e.g. TiBP 68\%, TnBP 93\%, TPhP $26 \%$, TBEP 39\%, TCrP 43\%, EHDPP 60\% and TEHP 27\% (Table S6).

In the activated sludge treatment system, the mass flows in both aqueous and solid phase through the anaerobic and anoxic tanks significantly increased, which was mainly resulted from the large quantity recycling of activated sludge with high mass flow of OPEs from the return sludge and internal recirculation sludge. It was worth noting that the solid phase mass flow percent of anaerobic tank increased to $52 \%$ from $7 \%$ in primary effluent, which was owing to the incoming of return sludge with a high solid phase mass flow percentage of 69\% (Table S4). Within the $\mathrm{A} / \mathrm{A} / \mathrm{O}$ treatment, the total mass flow loss percentages combined aqueous phase with solid phase were $-11 \%, 21 \%$, and $-13 \%$ in anaerobic, anoxic, and aerobic treatment units, respectively (Table 1). The efficiency of anoxic treatment was better than that of anaerobic and aerobic treatment. The mass flow loss percentages in aqueous and solid phases were individually 39\% and 7\% through the secondary treatment units (Table S7).

For the secondary effluent, the aqueous-phase mass flow of OPEs were $172 \pm 40 \mathrm{~g} / \mathrm{day}$, accounting for $53.8 \%$ of initial raw sewage mass flow, while the adsorbed part was negligible owing to the few content of suspended solids in secondary effluent. The traditional treatment removed about $38.4 \%$ of the initial OPEs mass flow from the aqueous phase (Table 2), indicating that, to a certain degree, the activated sludge system was effective to remove OPEs from sewage water by biosorption and biodegradation. Without further digestion, the excess sludge was then dehydrated to dewatered sludge with a total mass flow of OPEs $20 \pm 3.7 \mathrm{~g} /$ day during the three sampling days.

For advanced treatment, the OPEs mass flow over ultrafiltration showed no remarkable decrease for the amount of suspended solids in secondary effluent was little. After the ozonation treatment, the mass flow of OPEs was slightly increased, which could be attributed to their potential variations during the three sampling days. On the contrast, the OPEs mass flow after chlorination process was slightly decreased, but the removal efficiency of OPEs by chlorination was not so noticeable. 
Table 2

Comparison of measured OPEs by the current study with literature values reported in some developed countries and other regions of China.

\begin{tabular}{|c|c|c|c|c|c|c|c|c|c|c|c|c|c|c|c|}
\hline \multirow[t]{2}{*}{ OPES } & \multirow{2}{*}{$\begin{array}{l}\text { Log } \\
K_{\mathrm{OW}^{\mathrm{a}}}\end{array}$} & \multirow{2}{*}{$\begin{array}{l}\log \\
K_{\mathrm{OC}}{ }^{b}\end{array}$} & \multicolumn{6}{|c|}{ Current study } & \multirow{2}{*}{$\frac{\text { Spain }}{\text { Influent }^{\mathrm{e}}}$} & \multicolumn{2}{|l|}{ Germany } & \multirow{2}{*}{$\begin{array}{l}\text { Austria } \\
\text { Effluent }^{\mathrm{h}} \\
(\mathrm{ng} / \mathrm{L})\end{array}$} & \multicolumn{2}{|l|}{ Sweden } & \multirow{2}{*}{$\begin{array}{l}\text { China } \\
\text { Sludge } \\
\text { (ng/g, d.w.) }\end{array}$} \\
\hline & & & $\begin{array}{l}\text { Influent } \\
(\mathrm{ng} / \mathrm{L})\end{array}$ & $\begin{array}{l}\text { Secondary } \\
\text { effluent } \\
\text { (ng/L) }\end{array}$ & $\begin{array}{l}\text { Tertiary } \\
\text { effluent } \\
(\mathrm{ng} / \mathrm{L})\end{array}$ & $\begin{array}{l}\mathrm{E}_{\text {total }}{ }^{\mathrm{C}} \\
(\%)\end{array}$ & $\begin{array}{l}\mathrm{E}_{\mathrm{TT}}^{\mathrm{d}} \\
(\%)\end{array}$ & $\begin{array}{l}\text { Raw sludge } \\
\text { (ng/g, d.w.) }\end{array}$ & & $\begin{array}{l}\text { Influent }^{\mathrm{f}} \\
(\mathrm{ng} / \mathrm{L})\end{array}$ & $\begin{array}{l}\text { Sludge } \\
\text { (ng/g, d.w.) }\end{array}$ & & $\begin{array}{l}\text { Influent } \\
(\mathrm{ng} / \mathrm{L})\end{array}$ & $\begin{array}{l}\text { Sludge } \\
\text { (ng/g) }\end{array}$ & \\
\hline TMP & -0.65 & 1.10 & 99.2 & 75.9 & 67.2 & 32.2 & 23.5 & ND & NM & NM & NM & NM & $0-584$ & NM & NM \\
\hline TEP & 0.80 & 1.68 & 113.8 & 88.3 & 79.0 & 30.6 & 22.4 & ND & NM & NM & NM & $22-210$ & NM & NM & NM \\
\hline TCEP & 1.44 & 2.48 & 179.1 & 213.0 & 232.9 & -30.1 & -18.9 & ND & $0-70$ & $290-640$ & 70 & $0-1600$ & $90-1000$ & $6.6-110$ & NM \\
\hline TPrP & 1.87 & 2.83 & ND & ND & ND & - & - & ND & NM & NM & NM & NM & ND & NM & NM \\
\hline TCPP & 2.59 & 2.71 & 225.0 & 324.1 & 338.9 & -50.6 & -44.0 & 69.5 & $290-540$ & $2000-5800$ & 18,400 & $270-1400$ & $1100-18,000$ & 61-1900 & $7.9-35$ \\
\hline TDCP & 3.65 & 2.35 & 22.8 & 14.2 & 15.9 & 29.9 & 37.7 & 9.7 & $0-100$ & $100-250$ & 90 & $19-1400$ & $210-450$ & $3.0-260$ & $12.6-64$ \\
\hline TiBP & 3.60 & 3.05 & 45.2 & 56.6 & 25.9 & 42.7 & -25.3 & 26.4 & $39-188$ & $840-2200$ & 100 & NM & NM & $27-2700$ & NM \\
\hline $\mathrm{TPhP}$ & 4.59 & 3.72 & 21.3 & 15.9 & 4.4 & 79.4 & 25.5 & 75.0 & ND & $81-290$ & 400 & $0-170$ & $76-290$ & $52-320$ & $16-63$ \\
\hline TnBP & 4.00 & 3.28 & 74.4 & 28.8 & 29.8 & 59.9 & 61.2 & 100.8 & $0-82$ & $260-5500$ & 90 & $0-810$ & $6600-52,000$ & $39-780$ & $7.1-44$ \\
\hline CDPP & 3.70 & - & ND & ND & ND & - & - & ND & NM & NM & NM & NM & NM & NM & NM \\
\hline TBEP & 3.75 & 4.38 & 600.3 & 44.1 & 39.9 & 93.3 & 92.6 & 105.9 & $680-3100$ & $3700-6100$ & NM & $13-5400$ & $5200-35,000$ & $0-1900$ & $26-169$ \\
\hline TCrP & 5.11 & 4.35 & 8.7 & 2.0 & 0.4 & 95.2 & 77.3 & 87.8 & NM & NM & NM & $0-55$ & NM & NM & $3.8-14$ \\
\hline EHDPP & 6.64 & - & 14.0 & 0.8 & 0.9 & 93.9 & 94.1 & 56.7 & NM & NM & NM & NM & NM & $320-4600$ & NM \\
\hline TEHP & 9.49 & 6.87 & ND & ND & ND & - & - & 21.0 & NM & NM & NM & ND & $0-130$ & NM & NM \\
\hline$\sum$ OPES & & & 1399.0 & 862.4 & 832.9 & 40.5 & 38.4 & 552.7 & $1125.6-3994$ & $8720-20,420$ & 19,150 & $342-7777$ & $17,000-69,000$ & $620-6900$ & $101-290$ \\
\hline
\end{tabular}

- = data not available; $\mathrm{ND}=$ not detected (below method detection limit); $\mathrm{NM}=$ not measured.

${ }^{a}$ Reference: Reemtsma et al. (2008).

beference: van der Veen and de Boer (2012).

Removal efficiency of total treatment.

Removal efficiency of traditional treatment (combined of primary and secondary treatment)

Reference: Garcia-Lopez et al. (2010)

Reference: Meyer and Bester (2004).

Reference: Chen and Bester (2009).

Reference: Martinez-Carballo et al. (2007).

Reference: Marklund et al. (2005).

Reference: Zeng et al. (2014) (domestic wastewater in the Pearl River Delta, China). 


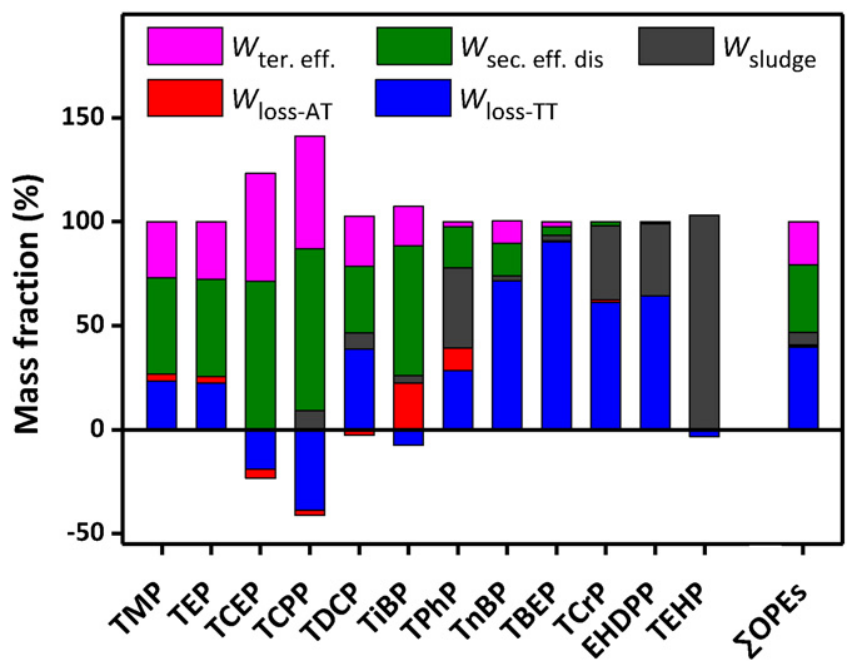

Fig. 3. Mass balance results: mass fraction (\%) of detected individual OPE and total OPEs ( $\sum$ OPEs $)$ in(i) tertiary effluents ( $W_{\text {ter. eff. }}$ ), (ii) secondary effluent discharged into the river $\left(W_{\text {sec. eff. dis. }}\right)$, (iii) dewatered sludge $\left(W_{\text {sludge }}\right)$, (iv) loss in advanced treatment $\left(W_{\text {loss-AT }}\right)$, and $(v)$ loss in traditional treatment $\left(W_{\text {loss-TT }}\right)$ relative to total initial loading (100\%) in the STP.

It was suggested that, the capability of these three advanced treatment units for removing the remaining OPEs in secondary effluent, which were mainly composed of TCEP and TCPP, was limited.

\subsection{Mass balance analysis of OPEs during wastewater treatment}

To analyze the fate of OPEs during wastewater treatment, mass balance calculation was performed based on the mass flow through the STP (Fig. 3). Total mass flow in raw sewage, including dissolved and adsorbed phases, was taken as the system input (100\%), while the system output consisted of (i) tertiary effluents, (ii) secondary effluent discharged to the river, (iii) dewatered sludge, (iv) loss in advanced treatment, and $(\mathrm{v})$ loss in traditional treatment including the primary and the secondary treatment, due to the total effect of degradation, transformation, and adsorption in each treatment unit in the STP.

The mass fraction of $\sum$ OPEs in secondary effluent discharged to the river and tertiary effluent accounted for $32.2 \pm 2.5 \%$ and $20.9 \pm 1.6 \%$ of the initial loading, respectively, and only $6.3 \pm 0.3 \%$ of the total amount of OPEs was transferred to dewatered sludge, while $39.9 \pm 4.4 \%$ of them was lost due to biodegradation. The mass loss fraction in advanced treatment was only $0.7 \pm 0.7 \%$, indicating the removal capacity of advanced treatment was negligible for OPEs with relatively high polarity (TEP, TCPP, TCEP), as most of the low polar OPEs (TBEP, TCrP, EHDPP, TEHP) were transformed or transferred during traditional treatment (Fig. 3).

For individual OPE, the behavior and fate showed a polarity-specific feature. OPEs with relatively high polarity ( $n$-octanol-water partition coefficients, $K_{\mathrm{OW}}<3$ ), including TMP, TEP, TCEP, and TCPP, showed no significant removal during both conventional treatment and advanced treatment, given that their mass flow loss percentages in each treatment units were lower than 20\%, except for that of TMP in ozonation unit (27\%) and TCPP in anoxic unit (35\%) (Table 1). As a result, these OPEs were mainly discharged along with the effluent. In contrast, for OPEs with medium polarity $\left(3<K_{\mathrm{OW}} \leq 4\right)$, alkyl TnBP and TBEP were much easier to be degraded by activated sludge with a mass loss rate of $71 \%$ and $90 \%$ in secondary treatment, respectively; while most of TiBP (81\%) and TDCP (56\%) transferred to the effluent, except a small part of TiBP (22\%) and TDCP (39\%) were individually lost in advanced treatment and traditional treatment. Meanwhile, aryl OPEs with relatively lower polarity $\left(4.5<K_{\mathrm{OW}}<7\right)$, including TPhP, TCrP and EHDPP, were mainly degraded by microorganism (29\%-64\%) and adsorbed by activated sludge (35\%-38\%). In addition, alkyl TEHP with the lowest polarity $\left(K_{\mathrm{OW}}=9.5\right)$ was mainly adsorbed by the activated sludge and showed no obvious biodegradation (Fig. 3).

It was noteworthy that there were negative mass fractions for several OPEs, especially for TCPP and TCEP (Fig. 3). The possible reason reported in literatures was that TCPP and TCEP included in polymeric particles of raw sewage may be released into the water phase during the sewage treatment, resulting the increased concentrations in effluent water compared to influent water (Bester, 2007). However, in present study, TCEP was not detected in solids phase of raw sewage (Fig. S2), and the mass flow of TCPP (Fig. S3) in solid phase of raw sewage (5.1

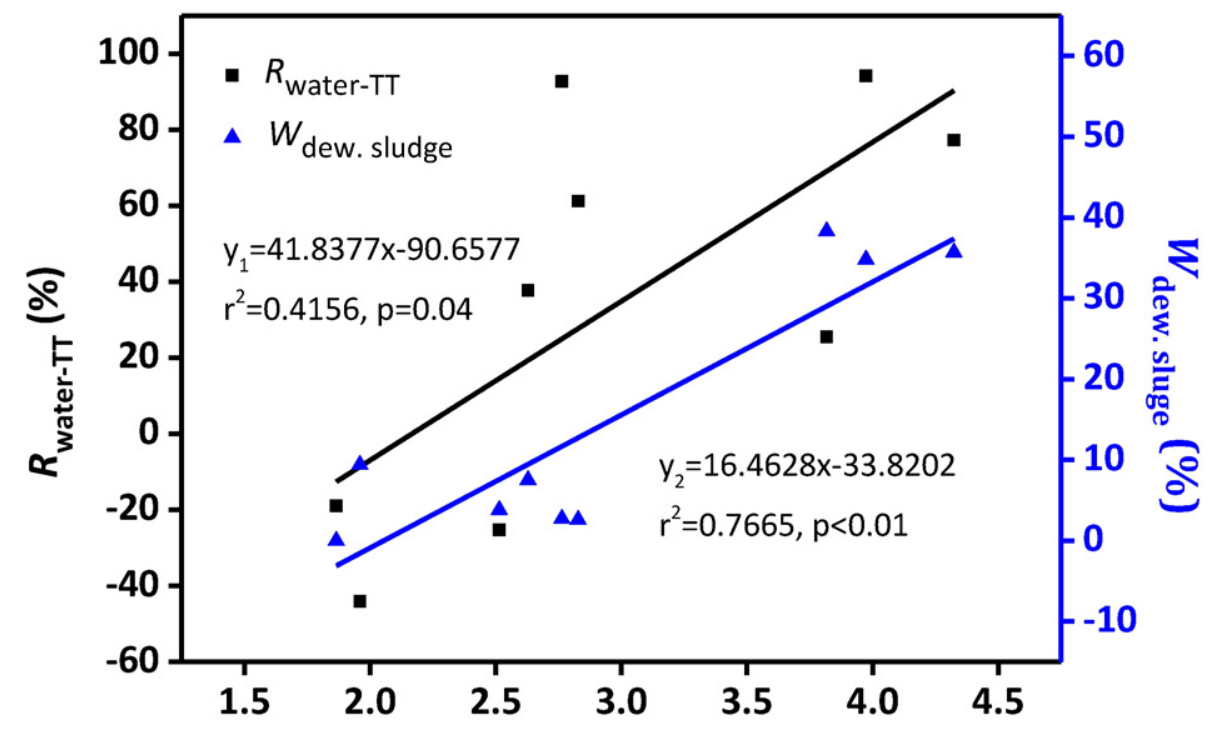

\section{$\log K_{\mathrm{d}}$}

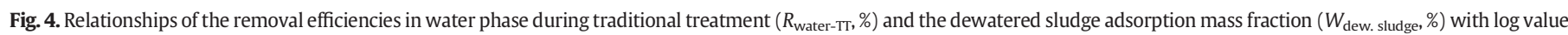
of apparent solid-water partition coefficients $\left(\log K_{\mathrm{d}}\right)$ of detected OPEs in the STP. 
g/day) was almost equivalent to that of dewatered sludge ( $4.7 \mathrm{~g} /$ day), indicating the exist of other major sources rather than polymeric particles. The possible sources for the increased concentration in effluent water were plastic tubes and ultrafiltration membranes used in the treatment procedure.

\subsection{Understanding the behavior and fate of OPEs during wastewater treatment}

To further understand the polarity-specific behavior and fate of the individual OPE, the apparent solid-water partition coefficients $\left(K_{\mathrm{d}}\right)$ of different OPEs between solid phase and aqueous phase were calculated (Supporting information, Table S8). As shown in Fig. S1, the $K_{\mathrm{d}}$ values of detected OPEs are comparable to their $K_{\mathrm{OW}}$ and organic carbon partition coefficients $\left(K_{\mathrm{OC}}\right)$ (Reemtsma et al., 2008; van der Veen and de Boer, 2012). They significantly increased with carbon atom numbers, which was consistent with variations of polarity-specific behavior and fate in the STP. On the other hand, the $K_{\mathrm{d}}$ values of most hydrophobic OPEs also significantly increased along the anaerobic-anoxic-aerobic treatment process (Table S8), suggesting that sorption to sludge particles was an important removal mechanism for most hydrophobic OPEs.

The average $\log K_{\mathrm{d}}$ values of detected OPEs (Table S8) showed a significant positive correlation with their dewatered sludge adsorption mass fraction ( $\left.W_{\text {sludge }}\right)\left(r^{2}=0.77, p<0.01\right.$, Fig. 4$)$, suggesting that hydrophobic interactions resulted in their adsorption to suspended solids. OPEs with higher $K_{d}$ values showed higher removal from the aqueous phase by activated sludge adsorption. This can explain why the average $\log K_{\mathrm{d}}$ values of detected OPEs also showed a significant positive correlation with their removal efficiencies of water phase during traditional treatment $\left(R_{\text {water-TT }}\right)\left(\mathrm{r}^{2}=0.42, \mathrm{p}=0.04\right.$, Fig. 4$)$.

In addition to suspended solid particles adsorption, biodegradation in activated sludge system also played an important role for the removal of OPEs in sewage (Fig. 3), but the degradation for individual OPE was very different from each other. Although hydrophobic OPEs are inclined to present in sludge where they have more opportunity to contact with zoogloea, the average $\log K_{\mathrm{d}}$ values of detected OPEs presented a relatively weak correlation with the mass loss fraction in traditional treatment $\left(W_{\text {loss-TT }}\right)\left(r^{2}=0.29, p=0.08\right.$, Fig. S4), suggesting hydrophobicity was not the primary factor leading to the different degradation degree for OPEs. Nevertheless, the degradation differences of OPEs in activated sludge treatment was in accordance with some general 'rules of thumb' based on their structural factors (Lyman et al., 1982): (i) OPEs with short chains (TMP, TEP) were not as quickly degraded as those with long chains (TnBP, TBEP), whereas they were less resistance to biodegradation than similar OPEs with chlorinated hydrocarbon chains (TCEP and TCPP); (ii) TiBP with branched hydrocarbon chains was less amenable to biodegradation compared to its unbranched isomer TnBP, while TEHP with longer branched hydrocarbon chains showed no obvious degradation; (iii) TBEP with 6 C hydrocarbon chains was easier to be biodegraded than OPEs with phenyl or tolyl group (TPhP, TCrP and EHDPP); (iv) Among the three aryl OPEs, EHDPP with 2 rings was more biodegradable than the two other compounds with 3 rings (TPhP and TCrP); and (v) owing to the electron donor property of aromatic substituent methyl, the biodegradation of TCrP was largely promoted compared to TPhP.

\section{Conclusion}

In summary, our work provides an insight into the behavior and the fate of OPEs in an advanced municipal STP based on mass flow and mass balance analysis. Sorption to activated sludge, which was proved to be dominated by hydrophobic interactions, played an important role in sewage treatment for OPEs. Meanwhile, biodegradation behaviors of individual OPE show a structure-specific feature. Hydrophobic OPEs in raw sewage with long hydrocarbon chains and aromatic groups were largely biodegraded by activated sludge treatment and partly adsorbed by suspended solid particles, with the remaining limited part transferring to the sewage effluent. Meanwhile, preferred to stay in water phase, hydrophilic OPEs with short non-chlorinated/chlorinated hydrocarbon chains were mostly discharged along with sewage effluent, with only a limited fraction biodegraded or transferred to the dewatered sludge during the activated sludge treatment. In this way, the different behaviors and distinct fate of individual OPE were presented in this municipal STP.

\section{Acknowledgments}

This work was financially supported by the National High Technology Research and Development Program of China (2013AA065201), and Chinese Academy of Sciences (XDB14020101, YSW2013A01, YSW2013B01).

\section{Appendix A. Supplementary data}

Supplementary data to this article can be found online at http://dx. doi.org/10.1016/j.scitotenv.2015.11.112.

\section{References}

Bester, K., 2005. Comparison of TCPP concentrations in sludge and wastewater in a typical German sewage treatment plant - comparison of sewage sludge from 20 plants. J. Environ. Monit. 7, 509-513.

Bester, K., 2007. Personal Care Compounds in the Environment: Pathways, Fate and Methods for Determination. John Wiley \& Sons.

Chen, X.J., Bester, K., 2009. Determination of organic micro-pollutants such as personal care products, plasticizers and flame retardants in sludge. Anal. Bioanal. Chem. 395, $1877-1884$

Cristale, J., Lacorte, S., 2013. Development and validation of a multiresidue method for the analysis of polybrominated diphenyl ethers, new brominated and organophosphorus flame retardants in sediment, sludge and dust. J. Chromatogr. A 1305, 267-275.

Ellis, J., Shah, M., Kubachka, K.M., Caruso, J.A., 2007. Determination of organophosphorus fire retardants and plasticizers in wastewater samples using MAE-SPME with GCICPMS and GC-TOFMS detection. J. Environ. Monit. 9, 1329-1336.

Fries, E., Mihajlovic, I., 2011. Pollution of soils with organophosphorus flame retardants and plasticizers. J. Environ. Monit. 13, 2692-2694.

Fries, E., Puttmann, W., 2003. Monitoring of the three organophosphate esters TBP, TCEP and TBEP in river water and ground water (Oder, Germany). J. Environ. Monit. 5, 346-352.

Gao, L.H., Shi, Y.L., Li, W.H., Ren, W.L., Liu, J.M., Cai, Y.Q., 2015. Determination of organophosphate esters in water samples by mixed-mode liquid chromatography and tandem mass spectrometry. J. Sep. Sci. 38, 2193-2200.

Garcia-Lopez, M., Rodriguez, I., Cela, R., 2008. Evaluation of liquid-liquid microextraction using polypropylene microporous membranes for the determination of organophosphorus flame retardants and plasticizers in water samples. Anal. Chim. Acta 625, 145-153.

Garcia-Lopez, M., Rodriguez, I., Cela, R., 2010. Mixed-mode solid-phase extraction followed by liquid chromatography-tandem mass spectrometry for the determination of tri- and di-substituted organophosphorus species in water samples. J. Chromatogr. A $1217,1476-1484$

Kim, J.W., Isobe, T., Chang, K.H., Amano, A., Maneja, R.H., Zamora, P.B., Siringan, F.P., Tanabe, S., 2011. Levels and distribution of organophosphorus flame retardants and plasticizers in fishes from Manila Bay, the Philippines. Environ. Pollut. 159, 3653-3659.

Leung, A.O.W., Luksemburg, W.J., Wong, A.S., Wong, M.H., 2007. Spatial distribution of polybrominated diphenyl ethers and polychlorinated dibenzo-p-dioxins and dibenzofurans in soil and combusted residue at Guiyu, an electronic waste recycling site in southeast China. Environ. Sci. Technol. 41, 2730-2737.

Liang, K., Niu, Y.M., Liu, J.F., 2014. Determination of 14 organophosphate ester flame retardants in wastewater by UPLC-MS/MS. Environ. Chem. 33, 1681-1685 (in Chinese)

Lyman, W.J., Reehl, W.F., Rosenblatt, D.H., 1982. Handbook of Chemical Property Estimation Methods: Environmental Behavior of Organic Compounds. McGraw-Hill, New York.

Marklund, A., Andersson, B., Haglund, P., 2003. Screening of organophosphorus compounds and their distribution in various indoor environments. Chemosphere 53 , 1137-1146.

Marklund, A., Andersson, B., Haglund, P., 2005. Organophosphorus flame retardants and plasticizers in Swedish sewage treatment plants. Environ. Sci. Technol. 39, 7423-7429.

Martinez-Carballo, E., Gonzalez-Barreiro, C. Sitka, A., Scharf, S. Gans, O, 2007. Determination of selected organophosphate esters in the aquatic environment of Austria. Sci. Total Environ. 388, 290-299.

Meyer, J., Bester, K., 2004. Organophosphate flame retardants and plasticisers in wastewater treatment plants. J. Environ. Monit. 6, 599-605. 
Mihajlovic, I., Miloradov, M.V., Fries, E., 2011. Application of twisselmann extraction, SPME, and GC-MS to assess input sources for organophosphate esters into soil. Environ. Sci. Technol. 45, 2264-2269.

Moller, A., Xie, Z.Y., Caba, A., Sturm, R., Ebinghaus, R., 2011. Organophosphorus flame retardants and plasticizers in the atmosphere of the North Sea. Environ. Pollut. 159, 3660-3665.

Moller, A., Sturm, R., Xie, Z.Y., Cai, M.H., He, J.F., Ebinghaus, R., 2012. Organophosphorus flame retardants and plasticizers in airborne particles over the northern Pacific and Indian Ocean toward the polar regions: evidence for global occurrence. Environ. Sci. Technol. 46, 3127-3134.

Posner, S., 2011. Assessment of the Consumption of HBCDD in EPS and XPS in Conjunction with National Fire Requirements. p. 76.

Quintana, J.B., Reemtsma, T., 2006. Potential of membrane-assisted solvent extraction for the determination of phosphoric acid triesters in wastewater samples by liquid chromatography-tandem mass spectrometry. J. Chromatogr. A 1124, 22-28.

Reemtsma, T., Quintana, J.B., Rodil, R., Garcia-Lopez, M., Rodriguez, I., 2008. Organophosphorus flame retardants and plasticizers in water and air I. Occurrence and fate. Tractrend. Anal. Chem. 27, 727-737.

Reemtsma, T., Lingott, J., Roegler, S., 2011. Determination of 14 monoalkyl phosphates, dialkyl phosphates and dialkyl thiophosphates by LC-MS/MS in human urinary samples. Sci. Total Environ. 409, 1990-1993.

Retardants-Online, F. Flame retardants-online. http://www.flameretardants-online.com (accessed on Augest 2015).

Rodil, R., Quintana, J.B., Reemtsma, T., 2005. Liquid chromatography-tandem mass spectrometry determination of nonionic organophosphorus flame retardants and plasticizers in wastewater samples. Anal. Chem. 77, 3083-3089.

Rodriguez, I., Calvo, F., Quintana, J.B., Rubi, E., Rodil, R., Cela, R., 2006. Suitability of solidphase microextraction for the determination of organophosphate flame retardants and plasticizers in water samples. J. Chromatogr. A 1108, 158-165.
Schindler, B.K., Forster, K., Angerer, J., 2009. Quantification of two urinary metabolites of organophosphorus flame retardants by solid-phase extraction and gas chromatography-tandem mass spectrometry. Anal. Bioanal. Chem. 395, 1167-1171.

Sundkvist, A.M., Olofsson, U., Haglund, P., 2010. Organophosphorus flame retardants and plasticizers in marine and fresh water biota and in human milk. J. Environ. Monit. 12, 943-951.

van den Eede, N., Dirtu, A.C., Ali, N., Neels, H., Covaci, A., 2012. Multi-residue method for the determination of brominated and organophosphate flame retardants in indoor dust. Talanta 89, 292-300.

van den Eede, N., Neels, H., Jorens, P.G., Covaci, A., 2013. Analysis of organophosphate flame retardant diester metabolites in human urine by liquid chromatography electrospray ionisation tandem mass spectrometry. J. Chromatogr. A 1303, 48-53.

van der Veen, I., de Boer, J., 2012. Phosphorus flame retardants: properties, production, environmental occurrence, toxicity and analysis. Chemosphere 88, 1119-1153.

Wang, X.W., Liu, J.F., Yin, Y.G., 2010. The pollution status and research progress on organophosphate ester flame retardants. Prog. Chem. 22, 1983-1992.

Wang, X.W., Liu, J.F., Yin, Y.G., 2011. Development of an ultra-high-performance liquid chromatography-tandem mass spectrometry method for high throughput determination of organophosphorus flame retardants in environmental water. J. Chromatogr. A 1218, 6705-6711.

Zeng, X., He, L., Cao, S., Ma, S., Yu, Z., Gui, H., Sheng, G., Fu, J., 2014. Occurrence and distribution of organophosphate flame retardants/plasticizers in wastewater treatment plant sludges from the Pearl River Delta, China. Environ. Toxicol. Chem. 33, 1720-1725. 